\title{
Long Term Outcome of Intravitreal Therapy with Pegaptanib Sodium in an Infant with Active Retinitis due to Postnatally Acquired Cytomegalovirus Infection
}

\author{
Cinzia Auriti ${ }^{1}$, Fiammetta Piersigillii, ${ }^{1, *}$, Gino Catena ${ }^{2}$ \\ ${ }^{1}$ Department of Medical and Surgical Neonatology, Bambino Gesu' Chldren’s Hospital (IRCCS) Rome, Italy \\ ${ }^{2}$ Department of Ophtalmology, Bambino Gesu' Chldren’s Hospital (IRCCS) Rome, Italy \\ *Corresponding author: fiammetta.piersigilli@opbg.net
}

Received March 30, 2015; Revised June 08, 2015; Accepted June 11, 2015

\begin{abstract}
We describe the case of an infant with a retinitis due to a postnatally acquired Cytomegalovirus, successfully treated at six months of life with three cycles of intravitreal injections of pegaptanib sodium. The therapy was effective in modulating the retinal disease activity in this immunocompetent child, without any side effect observed at a long-term follow up.
\end{abstract}

Keywords: cytomegalovirus infection, neonatal retinitis, newborn infant, Pegaptanib sodium

Cite This Article: Cinzia Auriti, Fiammetta Piersigilli, and Gino Catena, "Long Term Outcome of Intravitreal Therapy with Pegaptanib Sodium in an Infant with Active Retinitis due to Postnatally Acquired Cytomegalovirus Infection.” American Journal of Medical Case Reports, vol. 3, no. 7 (2015): 209-211. doi: 10.12691/ajmcr-3-7-7.

\section{Case Report}

A $2850 \mathrm{~g}$ boy spontaneously delivered at 38 weeks of gestational age was hospitalized at 20 days of life for anemia, poor feeding and failure to thrive. Because of symptomatic anemiahe was transfused with $20 \mathrm{ml} / \mathrm{Kg}$ packed red blood cells. A few days later, Cytomegalovirus (CMV) infection was diagnosed. Maternal serologic CMV tests during pregnancy showed high-avidity IgG (without seroconversion) and no IgM antibodies. Polymerase Chain Reaction (PCR) viral DNA analysis performed on maternal urine, saliva and milk was negative. Therefore, CMV infection was considered as postnatally acquired. We retrospectively performed a CMV-DNA analysis (artus CMV TM PCR kit, Qiagen, according to the TaqMan real-time PCR technique and using the ABI PRISM 7900 Sequence Detection System, Applied Biosystem, Foster City, CA, USA), on a $40 \mu$ dried blood spot of the neonatal screening card collected at three days of life. The resultwas negative, further confirming the hypothesis of a postnatally acquired infection.

At 28 days of life fundus examination showed hemorrhage and partial retinal detachment in the left eye; 5 days later complete retinal detachment occurred. The ocular magnetic resonance and ultrasound scan confirmed complete retinal detachment, with exudates and vitreitis in the left eye and sub retinal and intravitreal hemorrhages even in the right eye. Other causes of infectious retinitis were excluded (serologic tests for Human Immunodeficiency Virus, Toxoplasma gondii, Rubella virus, Herpes viruses 1 and 2 and Epstein Barr Virus were
negative).Flow cytometry study of lymphocyte subsets ( $\mathrm{T}$ Limphocytes CD4+ and CD8+, B lymphocytes CD19+, NK cells CD 16+56+, IgG, IgA and IgM) yielded normal findings. After two weeks of intravenous therapy with ganciclovir, $10 \mathrm{mg} / \mathrm{kg} / \mathrm{day}$, hemorrhage and exudation began to resolve in both eyes. After three weeks of therapy fundus examination showed initial scarring in the left eye and resolution of the lesions in the right eye, therefore iv therapy was stopped. Oral valganciclovir 30 $\mathrm{mg} / \mathrm{kg} /$ day for one week was further administered. CMV DNA load in whole blood was negative at discontinuation of therapy $[1,2,3]$.

At 5 months of life the baby presented fever and neutropenia (WBC=5,820/mm3, neutrophils absolute number $830 / \mathrm{mm} 3$ ) and he was rehospitalized. The PCR on plasma and blood showed CMV reactivation. We started intravenous Gancyclovir with resolution of the acute symptoms after two weeks of therapy. Angiography can highlight underlying or active vasculopathy by showing occlusive events, neovascularization or hemorrhage. To better study a possible reactivation of the retinitis we therefore performed an angiography that showed a subretinalneovascular area of proliferation in the left eye, with neuroepithelial detachment. At 6 months of life parental written consensus was obtained and the child underwent 3 intravitreal injections (1 every 8 weeks) with $1,65 \mathrm{mg}$ pegaptanib sodium per dose (Macugen ${ }^{\circledR}$ Pfizer Company), corresponding to $0,3 \mathrm{mg}$ of the free acid form of the oligonucleotide (corresponding to a volume of 90 microliters). Each injection was performed in the operating theatre, under general anesthesia, after having obtained digital wide field camera exam (Retcam 120) and fluorangiography of both eyes. The drug was injected at 
2,5 $\mathrm{mm}$ from the sclerocorneallimbus, by means of a $27 \mathrm{G}$ needle. The left eye was medicated daily with antibiotic drops and was blindfolded; amoxicillin $50 \mathrm{mg} / \mathrm{Kg} /$ day was orally administered for 5 days. We observed a progressive reduction of the neovascular membrane and the development of a progressive chorioretinal fibrous scar (Figure 1). The baby did not present any of the local side effects reported in the literature, namelysubconjuntival hemorrhage, lens rupture, ocular hypertonia, vitreous hemorrhage, endophtalmitis. The test for CMV DNA by PCR performed on whole blood and plasma remained negative. The visual evoked neural potential control on the left eye showed a well structured signal amplitude and a normal latency. At 8 months of life visual evoked potentials showed left prechiasmatic visual pathway

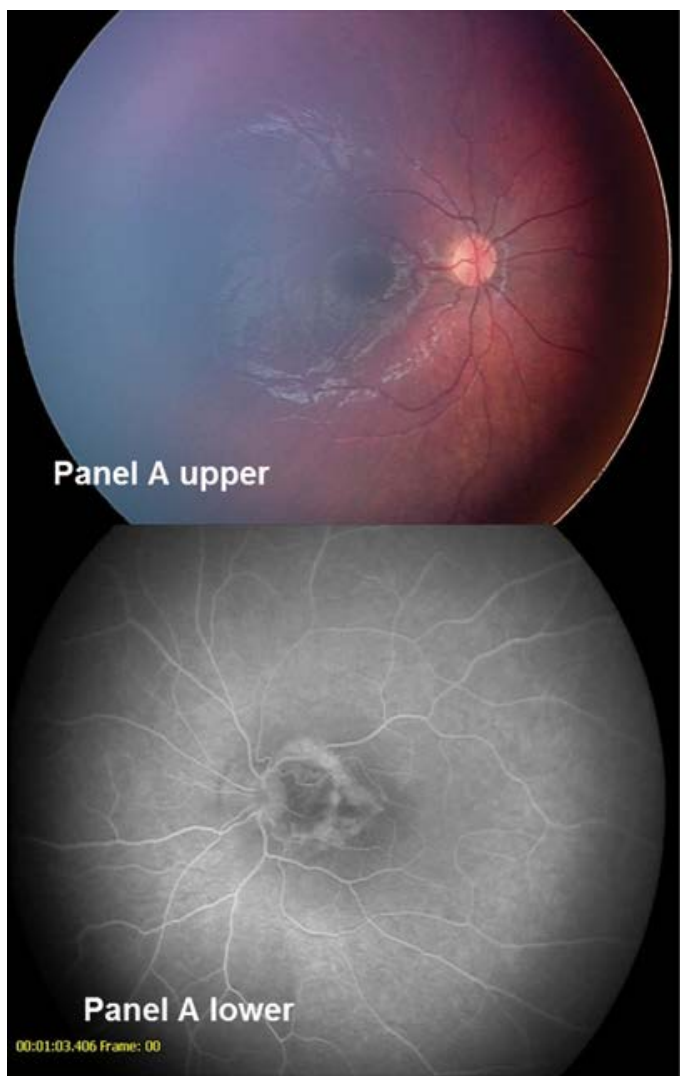

impairment whereas electroretinography yielded normal findings. At last follow up performed at 6 years of age the child was wearing glasses. Best Corrected Visual Acuity (BCVA) was 20/200 in the right eye and 20/20 in the left eye. Cycloplegic refraction, obtained after instillation of 1 drop of cyclopentolate, showedemmetropy in the right eye and myopia (-4D) in the left eye. The ocular motility test demonstrated incomitantesotropy in the left eye. Fund us examination of the left eye showed a peripapillarychorioretinal pigmented lesion touching the macular area with an overhanging veil of epiretinal gliosis. The last fluorangiography, performed at 5 years of age, highlighted atrophic macular post-infectious choroidal neovascularization (CNV) located between the papilla and the macula, with partial involvement of the central area.

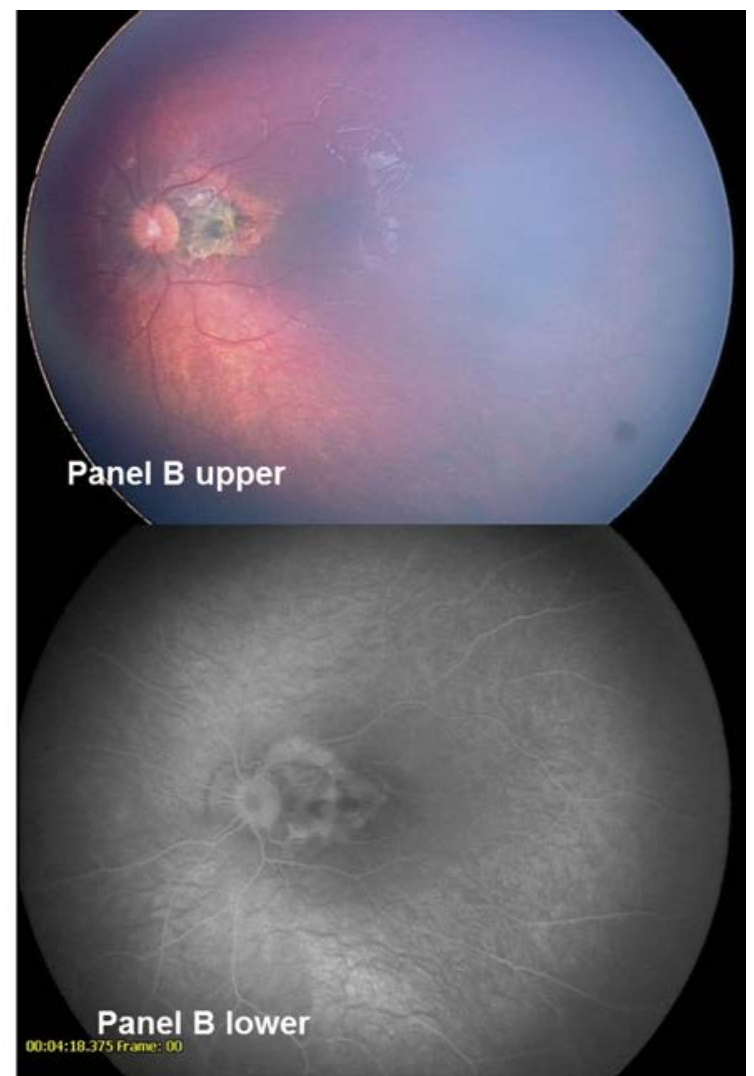

Figure 1. Digital wide field-camera (Retcam 120) images (upper part of the figure) and retinal fluorangiography (lower part of the figure) (panel A) before and after (panel B) pegaptanib sodium therapy. Of note the progressive reduction of the neovascular membrane and outcome in chorioretinal fibrous scar showed in the Retcam images

\section{Discussion}

At the time of treatment only two oligonucleotide drugs had been approved for clinical use. The first drug available for treating CMV-induced retinitis was a phosphorothioate antisense olinucleotide (fomivirsen), which inhibits CMV replication by an antisense mechanism [4]. The second drug was a PEGylate RNA aptamer, a conjugate of an aptamer (pegaptanib), directed against vascular endothelial growth factor, isoform 165 (VEGF-165), responsible for neovascularization and vascular permeability. The latter drug has been used for treating neovascular age-related macular degeneration (AMD) [5]. Since 2004 pegaptanib (Macugen; Eyetech Pharmaceuticals, New York, NY) has been successfully used in the treatment of all forms of AMD in adults [6,7]. However, despite repeated, high-cost treatment sessions of pegaptanib, patients generally lose vision over time because the growth of neovascular lesions is slowed, but not stopped. There is still little information about longterm outcomes of anti-VEGF treatment in children.

We presented the case of an infant with postnatal CMV infection and active retinitis, characterized by a subretinalneovascular area of proliferation, successfully treated with intravitreal anti-VEGF and followed up for 6 years. This therapy was effective in modulating the retinal disease activity in this immunocompetent infant. We observed regression of neovascularization and stability of the framework over time, as evidenced by the controls performed after the end of treatment and repeated every year by means of retinal fluorangiography. Further larger studies are required to assess the true value of this therapeutic approach in children. Nevertheless one must bear in mind that the most important challenge is to develop correct strategies for the prevention of horizontal 
transmission of CMV infection, particularly in neonates, who are at risk for developing life-threatening disease.

\section{Funds}

No grants or funds supported this study.

\section{References}

[1] Piersigilli F, Catena G, De Gasperis MR et al. Active retinitis in an infant with postnatally acquired cytomegalovirus infection. $J$ Perinatol. 32:559-62. 2012.
[2] Coors LE, Spencer R Delayed presentation of cytomegalovirus retinitis in an infant with severe congenital cytomegalovirus infection. Retina, (4 Suppl):S59-62. Apr; 2010

[3] Costagliola C, Romano MR, Parmeggiani F et al. Epiretinal membrane in a 12-year-old immunocompetent girl with cytomegalovirus infection. Eur J Ophthalmo, 19:1098-101. 2009.

[4] Crooke ST. Vitravene-another piece in the mosaic. Antisense Nucleic Acid Drug Dev, 8:vii-viii1998

[5] Ng EW, Shima DT, Calias P et al. Pegaptanib, a targeted antiVEGF aptamer for ocular vascular disease. Nat Rev Drug Discover, 5:123-132. 2006.

[6] Gragoudas ES, Adamis AP, Cunningham ET Jr, et al. Pegaptanib for neovascular age-related macular degeneration. $N$ Engl J Med, 351:2805-2816. 2004

[7] The Eyetech Study Group. Preclinical and phase 1A clinical evaluation of an anti-VEGF pegylatedaptamer (EYE001) for the treatment of exudative age-related macular degeneration. Retina, 22:143-152. 2002. 